ARTICLE

\title{
The E3 ubiquitin ligase TRIM31 attenuates NLRP3 inflammasome activation by promoting proteasomal degradation of NLRP3
}

Hui Song ${ }^{1,2}$, , Bingyu Liu ${ }^{1,2, \star}$, Wanwan Huai ${ }^{1,2, \star}$, Zhongxia Yu ${ }^{1,2}$, Wenwen Wang ${ }^{1,2}$, Jing Zhao ${ }^{1,2}$, Lihui Han ${ }^{1,2}$, Guosheng Jiang ${ }^{3}$, Lining Zhang ${ }^{1,2}$, Chengjiang Gao ${ }^{1,2} \&$ Wei Zhao ${ }^{1,2}$

The NLRP3 inflammasome has a fundamental role in host defence against microbial pathogens and its deregulation may cause diverse inflammatory diseases. NLRP3 protein expression is a rate-limiting step for inflammasome activation, thus its expression must be tightly controlled to maintain immune homeostasis and avoid detrimental effects. However, how NLRP3 expression is regulated remains largely unknown. In this study, we identify E3 ubiquitin ligase TRIM31 as a feedback suppressor of NLRP3 inflammasome. TRIM31 directly binds to NLRP3, promotes K48-linked polyubiquitination and proteasomal degradation of NLRP3. Consequently, TRIM31 deficiency enhances NLRP3 inflammasome activation and aggravates alum-induced peritonitis in vivo. Furthermore, TRIM31 deficiency attenuates the severity of dextran sodium sulfate (DSS)-induced colitis, an inflammatory bowel diseases model in which NLRP3 possesses protective roles. Thus, our research describes a mechanism by which TRIM31 limits NLRP3 inflammasome activity under physiological conditions and suggests TRIM31 as a potential therapeutic target for the intervention of NLRP3 inflammasome related diseases.

\footnotetext{
${ }^{1}$ Department of Immunology, Shandong University School of Medicine, Jinan, Shandong 250012, China. ${ }^{2}$ Key Laboratory of Infection and Immunity of Shandong Province, Shandong University School of Medicine, Jinan, Shandong 250012, China. ${ }^{3}$ Institute of Basic Medicine, Shandong Academy of Medical Sciences, Jingshi Road 18877, Jinan, Shandong 250062, China. * These authors contributed equally to this work. Correspondence and requests for materials should be addressed to C.G. (email: cgao@sdu.edu.cn) or to W.Z. (email: wzhao@sdu.edu.cn).
} 
$\mathrm{N}$ LRP3 inflammasome is a multi-protein platform which comprises NLRP3, ASC and caspase-1, and plays crucial roles in host defence against pathogens ${ }^{1-5}$. Inflammasome complex assembly is triggered by stimuli from both microbial infection and endogenous 'danger signal', such as nigericin, crystals, extracellular ATP, amyloid- $\beta$ and alum and so on. And then the inflammasome complex serves as platforms for the activation of the cysteine protease caspase-1, which cleaved pro-IL-1 $\beta$ and pro-IL-18 into mature IL-1 $\beta$ and IL-18 (refs $1-5$ ). NLRP3 inflammasome has been implicated in many kinds of diseases, such as gout, autoimmune disorders, atherosclerosis, type 2 diabetes and obesity ${ }^{5-9}$. Thus, NLRP3 inflammasome activity must be tightly controlled to maintain immune homeostasis and avoid detrimental effects.

NLRP3 expression level is considered as a limiting step in inflammasome activation ${ }^{10,11}$. In resting macrophages, the protein level of NLRP3 is relatively low, so that NLRP3 inflammasome assembly is hardly induced ${ }^{11,12}$. Induction of NLRP3 protein expression licensed by TLR ligands (signal 1) allows respective NLRP3 activators (signal 2) to trigger caspase-1 cleavage $^{10,13}$. Thus, regulation of NLRP3 level offers an interesting mechanism to alter the inflammatory potential of immune cells ${ }^{11}$. Up to now, several mechanisms for negative regulation of NLRP3 expression have been established. For example, we reported that aryl hydrocarbon receptor could bind to the NLRP3 promoter and inhibit its expression at transcriptional level ${ }^{14}$. MiR-223 suppresses NLRP3 mRNA expression through a conserved binding site within the $3^{\prime}$ untranslated region of NLRP3 (refs 15,16).

Autophagy-lysosomal pathway and ubiquitin-proteasome pathway are two major protein degradation systems widely exist in mammalian cells, which provide specificity and regulate the intensity of innate immune responses. It has been reported that autophagy-dependent degradation was involved in the regulation of NLRP3 expression ${ }^{17,18}$. For example, plasminogen activator inhibitor type 2 enhances NLRP3 degradation in an autophagy-dependent manner ${ }^{17}$. Dopamine D1 receptor (DRD1) signalling promotes NLRP3 ubiquitination via E3 ubiquitin ligase MARCH7, leading to the autophagy-mediated degradation of NLRP3 (ref. 18). However, whether ubiquitin-proteasome pathway is also involved in the regulation of NLRP3 protein expression remains unknown.

TRIM family proteins have been implicated in the negative regulation of innate immune responses by promoting degradation of their respective substrates through ubiquitin-proteasome pathway ${ }^{19}$. TRIM31 is a member of the TRIM protein family, which is encoded within the major histocompatibility complex (MHC) class I region ${ }^{20}$. Several TRIM family members (including TRIM31, TRIM40, TRIM10, TRIM15, TRIM26 and TRIM39) are organized in a tight cluster and two TRIM genes (including TRIM38 and TRIM27) telomeric of the cluster within the MHC class I region ${ }^{20}$. Most of the above mentioned TRIM family members possess vital regulatory effects in innate immune responses ${ }^{19,21-27}$. However, the biological function of TRIM31, especially in the innate immune response, remains unknown.

In this report, we describe a mechanism by which TRIM31 negatively regulates NLRP3 inflammasome activity. TRIM31 could directly bind to NLRP3, and then promote K48-linked polyubiquitination and proteasomal degradation of NLRP3 in both resting and activated macrophages. The identification of TRIM31 as a physiological suppressor of NLRP3 expression provides an explanation on the limitation of NLRP3 inflammasome activation under physiological conditions. Consistent with that, TRIM31 deficiency facilitated NLRP3 inflammasome activation, enhanced IL-1 $\beta$ secretion and thus aggravated alum-induced peritonitis in vivo. On the other hand, TRIM31 deficiency attenuated the severity of dextran sodium sulfate (DSS)-induced colitis by promoting NLRP3 inflammasome activation, which was reported to be possessing protective roles in the model. Thus, TRIM31 could be a potential therapeutic target for NLRP3-associated syndromes, including autoinflammatory and autoimmune diseases.

\section{Results}

TRIM31 specifically inhibits NLRP3 inflammasome activation. To investigate the potential functions of TRIM31 in innate immune response, specific and effective siRNA targeting TRIM31 was used to suppress the expression of endogenous TRIM31 in mouse macrophages (Supplementary Fig. 1a). TRIM31 knockdown has no regulatory effects on mRNA expression of lipopolysaccharide (LPS)-induced proinflammatory cytokines, including TNF- $\alpha$ and IL-1 $\beta$ (Supplementary Fig. $1 \mathrm{~b}$ ). But, IL-1 $\beta$ secretion was significantly increased in TRIM31 silenced macrophages primed by LPS and then treated by NLRP3 inflammasome activator such as ATP, nigericin or alum (Fig. 1a). However, TNF- $\alpha$ and IL- 6 secretion were not influenced by TRIM31 knockdown (Fig. 1a). Similarly, TRIM31 overexpression greatly inhibited IL-1 $\beta$ secretion in human THP-1 cells (Fig. 1b). To confirm the inhibitory role of TRIM 31 in IL- $1 \beta$ secretion, the effects of TRIM31 deficiency on IL-1 $\beta$ expression in mouse peritoneal macrophages were observed. ATP and Nig stimulated IL-1 $\beta$ secretion by LPS-primed TRIM31-deficient macrophages was significantly increased (Fig. 1c, Supplementary Fig. 1c-e). However, IL-1 $\beta$ secretion stimulated by poly(dA:dT) (an AIM2 inflammasome activator) and flagellin(a NLRC4 inflammasome activator) was not influenced by TRIM31 deficiency (Fig. 1c, Supplementary Fig. 1d,e). In addition, TRIM31 deficiency also enhanced ATP stimulated IL-1 $\beta$ secretion in PGN-primed macrophages (Supplementary Fig. 1f). Furthermore, TRIM31 deficiency also had no inhibitory effects on TNF- $\alpha$, IL-6 expression and IL-1 $\beta$ mRNA expression (Supplementary Fig. 1g). Taken together, these data indicated that TRIM31 specifically inhibited NLRP3 inflammasome activation.

Caspase- 1 cleavage is a critical step for the NLRP3 inflammasome activation. We then investigated the effects of TRIM31 on caspase-1 cleavage. TRIM31 knockdown and deficiency both significantly enhanced caspase-1 cleavage in LPS-primed mouse peritoneal macrophages treated by ATP (Fig. 1d,e). Accordingly, less cleaved caspase-1 was detected in TRIM31 overexpressed THP-1 cells (Fig. 1f). However, the TRIM31 truncated mutant $\triangle \mathrm{RING}$, in which the N-terminal RING domain was deleted and the potential E3 ubiquitin ligase activity was deprived, lost the ability to inhibit caspase-1 cleavage, compared with TRIM31 WT (Fig. 1f).

To further rule out the possible regulatory effects of TRIM31 in the priming process of NLRP3 inflammasome activation, we explored the function of TRIM31 on NF- $\kappa B$ and MAPK activation. TRIM31 knockdown had no influence on LPS-induced phosphorylation of I $\mathrm{KB}-\alpha$, JNK, ERK and p38 (Supplementary Fig. 1h). Additionally, no differences of MyD88- and TNF- $\alpha$ induced $\mathrm{NF}-\kappa \mathrm{B}$ reporter gene activation were observed in TRIM31 overexpressed HEK293T cells (Supplementary Fig. 1i). Collectively, these data indicated that TRIM31 specifically inhibited NLRP3 inflammasome activation and subsequent IL- $1 \beta$ secretion.

TRIM31 promotes proteasomal degradation of NLRP3. TRIM family proteins have been implicated in the negative regulation of innate immune responses by promoting degradation of their respective substrates through ubiquitin-proteasome pathway ${ }^{19}$. We next sought to determine whether TRIM31 could inhibit 
NLRP3 inflammasome activation by promoting protein degradation of inflammasome components. TRIM31 knockdown greatly increased NLRP3 protein expression in resting mouse peritoneal macrophages, with no influence on ASC and caspase-1 protein expression (Fig. 2a). Similarly, TRIM31 knockdown also significantly increased NLRP3 protein level in LPS-priming macrophages (Fig. 2b). But, TRIM31 knockdown had no effects on NLRP3 mRNA expression (Fig. 2a,b). Consistently, TRIM31 overexpression greatly decreased NLRP3 protein expression in a dose-dependent manner in HEK293T cells (Fig. 2c). Importantly, similar results were also observed in TRIM31 deficient mouse peritoneal macrophages. TRIM31 deficiency greatly enhanced NLRP3 protein expression in both resting and activated macrophages (Fig. 2d, Supplementary Fig. 2). But, TRIM31 deficiency could not increase AIM2 and NLRC4 expression (Fig. 2d). We next examined the effects of TRIM31 deficiency on other agonistsinduced NLRP3 expression. TRIM31 deficiency greatly enhanced IL-1 $\beta$-, poly(I:C)- and PGN-induced NLRP3 expression in macrophages (Fig. 2e). However, no marked difference of TNF$\alpha$-induced NLRP3 expression was observed in TRIM31 deficient macrophages (Fig. 2e).

Given the functions of TRIM family members in promoting the degradation of their target proteins, we inferred that TRIM31 might inhibit NLRP3 protein expression via promoting its degradation. TRIM31 deficiency inhibited NLRP3 protein degradation (Fig. 2f,g). However, no differences of ASC,
Caspase-1 and NLRC4 protein degradation were observed (Fig. 2f,g). These data indicated that TRIM31 inhibited NLRP3 expression via promoting its protein degradation. We then explored the degradation pathway of NLRP3 mediated by TRIM31. TRIM31-induced NLRP3 degradation could be reversed by proteasome inhibitor MG-132, but not by lysosome inhibitor chloroquine (Fig. 2h) or autophagy inhibitor 3-MA (Fig. 2i), indicating that TRIM31 mediated NLRP3 degradation in proteasome. Next, we examined the NLRP3 expression in both wild type (WT) and TRIM31-deficient macrophages following MG132 treatment. MG132 treatment slightly enhanced NLRP3 expression in unactivated WT macrophages, with no effects on LPS-primed WT macrophages (Fig. 2j). In MG132-treated macrophages, TRIM31 deficiency could not enhance NLRP3 expression (Fig. 2j). Interestingly, TRIM31 deficiency greatly inhibited NLRP3 expression in LPS-primed and MG132-treated macrophages (Fig. 2j). These data indicated that MG132, as a general proteosome inhibitor, could not markedly affect NLRP3 expression in WT macrophages. However, MG132 treatment could block or reverse the effects of TRIM31 deficiency on NLRP3 expression. Taken together, these results indicated that TRIM31 could promote proteasomal degradation of NLRP3.

TRIM31 interacts with NLRP3. To further investigate how TRIM31 promotes NLRP3 degradation, we first examined the interaction between TRIM31 and NLRP3. In vitro binding assays

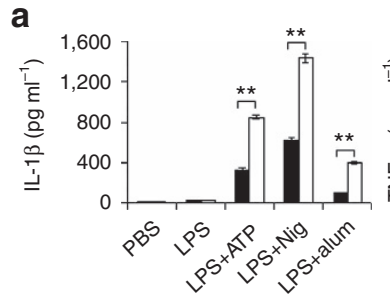

C

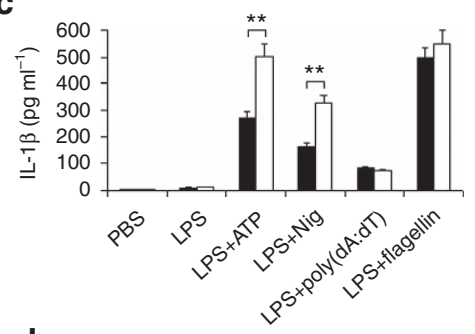

d

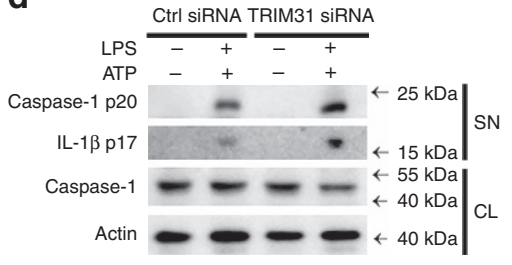

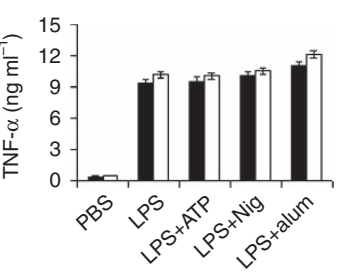

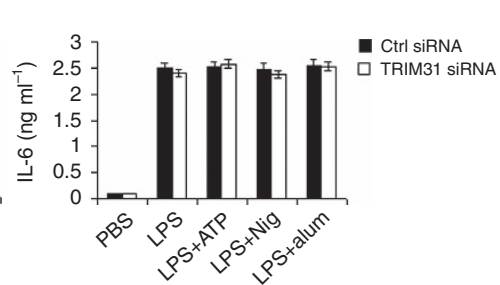

b
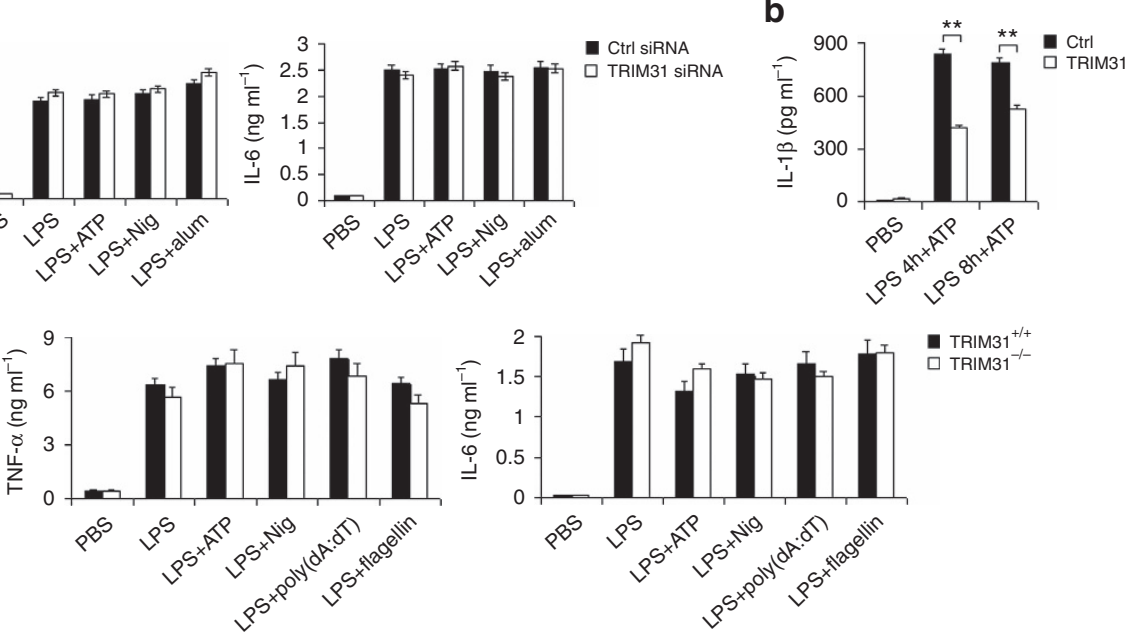

e

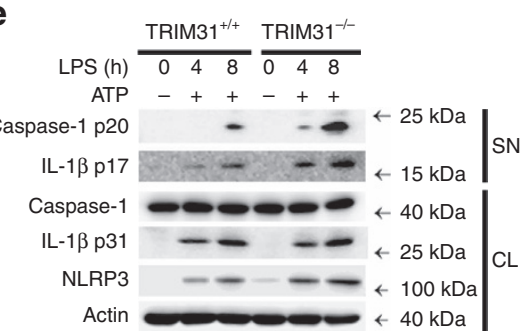

f

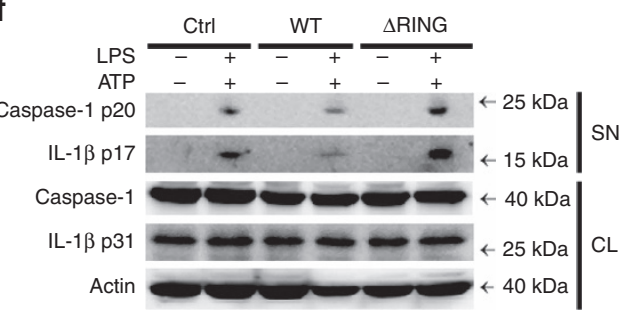

Figure 1 | TRIM31 specifically inhibits NLRP3 inflammasome activation. (a) ELISA of IL-1 $\beta$, TNF- $\alpha$ and IL- 6 in supernatants from mouse peritoneal macrophages silenced of TRIM31, primed with LPS for $8 \mathrm{~h}$, and followed by stimulation with ATP, Nig. or alum for 30 min. (b) ELISA of IL-1 $\beta$ in supernatants from THP-1 cells transfected with TRIM31 plasmid, primed with LPS for various times, and followed by stimulation with ATP for 30 min. (c) ELISA of IL-1 1 , TNF- $\alpha$ and IL- 6 in supernatants of mouse peritoneal macrophages from TRIM31+/+ or TRIM $31^{-/-}$mice, primed with LPS for $8 \mathrm{~h}$, and followed by stimulation with ATP, Nig., poly(dA:dT) or flagellin for 30 min. (d) Immunoblot analysis of supernatants (SN) or cell lysates (CL) from mouse peritoneal macrophages silenced of TRIM31, primed with LPS, and followed by stimulation with ATP for 30 min. (e) Immunoblot analysis of supernatants (SN) or cell lysates $(\mathrm{CL})$ of mouse peritoneal macrophages from TRIM31+/+ or TRIM31-/ - mice, primed with LPS, and followed by stimulation with ATP for 30 min. (f) Immunoblot analysis of supernatants (SN) or cell lysates (CL) from THP-1 transfected with TRIM31 WT or $\triangle$ RING mutant, primed with LPS, and followed by stimulation with ATP for $30 \mathrm{~min}$. Similar results were obtained in three independent experiments. ${ }^{\star \star} P<0.01$. (Student's $t$-test). Data are representative of three experiments (mean and s.d. of six samples in $\mathbf{a}-\mathbf{c}$ ). 
demonstrated that TRIM31 could directly interact with NLRP3 (Fig. 3a). And then, to confirm the interaction between TRIM31 and NLRP3 in vivo, we assessed resting and LPS-stimulated macrophages by immunoprecipitation (IP). An association between TRIM31 and NLRP3 could be detected in both mouse peritoneal macrophages and THP-1 cells (Fig. 3b, Supplementary Fig. 3a). To investigate whether TRIM31 interacted with other NLRP3 inflammasome molecules, we expressed TRIM31 with NLRP3, caspase-1 and ASC respectively, in HEK293T cells. TRIM31 could immunoprecipitate with NLRP3, and could not immunoprecipitate with caspase-1 and ASC (Fig. 3c, Supplementary Fig. 3b). Consistently, confocal analysis also demonstrated the colocalization between TRIM31 and NLRP3 (Fig. 3d, Supplementary Fig. 3c). Taken together, these data suggested that TRIM31 could directly interact with NLRP3.

TRIM31, as a member of the TRIM family proteins, contains an N-terminal RING-finger domain, B-box domain and two C-terminal coiled-coil (CC) domains (Fig. 3e). To search for the domains of TRIM31 that is responsible for the interaction with NLRP3, a series of Flag-tagged TRIM31 truncated mutants were constructed (Fig. 3e). NLRP3 was coprecipitated with TRIM31 WT, RING domain deletion mutant ( $\triangle$ RING), C126 and C270, but not with N131 and N162 (Fig. 3f). These results indicated that TRIM31 interacted with NLRP3 via its second C-terminal coiled-coil domain. Three truncated mutants of NLRP3 were also constructed (Fig. 3g). Co-IP experiments with these truncated mutants showed that NLRP3 $\triangle$ PYD lost the ability to bind
TRIM31, while NLRP3 $\triangle \mathrm{LRR}$ and $\triangle \mathrm{NACHT}$ did not (Fig. $3 \mathrm{~h}$ ). So the PYD domain of NLRP3 is required for the NLRP3-TRIM31 interaction.

TRIM31 promotes K48-linked polyubiquitination of NLRP3. Protein ubiquitination is a key step in the ubquitin-proteasome degradation pathway ${ }^{28}$. NLRP3 could be ubiquitinated with both K48 and K63 linkage in macrophages (Supplementary Fig. 4), and that is consistent with a previous report ${ }^{18}$. We have identified E3 ubiquitin ligase TRIM31 as a NLRP3-associated protein (Fig. 3) and that promoted us to investigate whether TRIM31 could mediate the ubiquitination of NLRP3. NLRP3 was cotransfected with HA-ubiquitin and WT TRIM31 into HEK293T cells. NLRP3 ubiquitination was markedly increased in the presence of TRIM31 expression plasmid (Fig. 4a). Importantly, the TRIM31 point mutation (C16AC36A) with substitution of the cysteine residue with alanine at position 16 and 36 within the RING domain, lost the ability to promote polyubiquitination of NLRP3 (Fig. 4a), indicating TRIM31 could promote the ubiquitination of NLRP3 though the N-terminal RING-finger domain. To study the forms of TRIM31-mediated NLRP3 polyubiquitination, ubiquitin mutant vectors K48 and K63, which contain arginine substitutions of all of its lysine residues except the one at position 48 and 63 respectively, were used in the transfection assays. TRIM31 promoted NLRP3 polyubiquitination could be detected in the presence of K48 plasmid, but not with K63 a

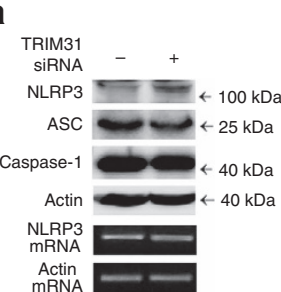

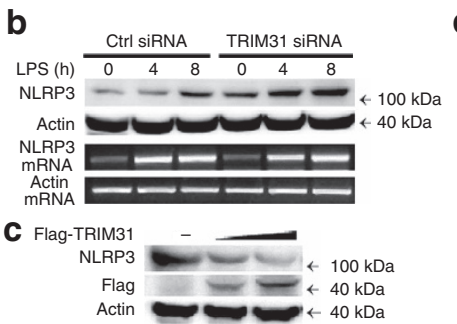

d

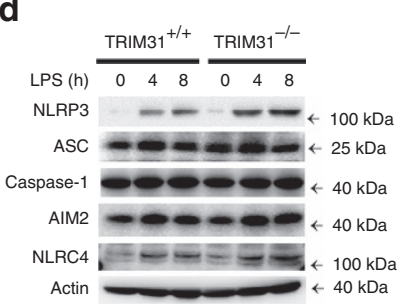

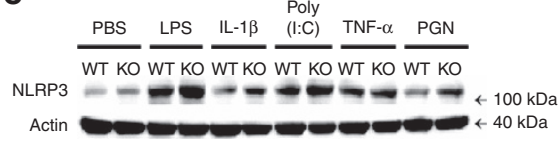

$\mathbf{f}$
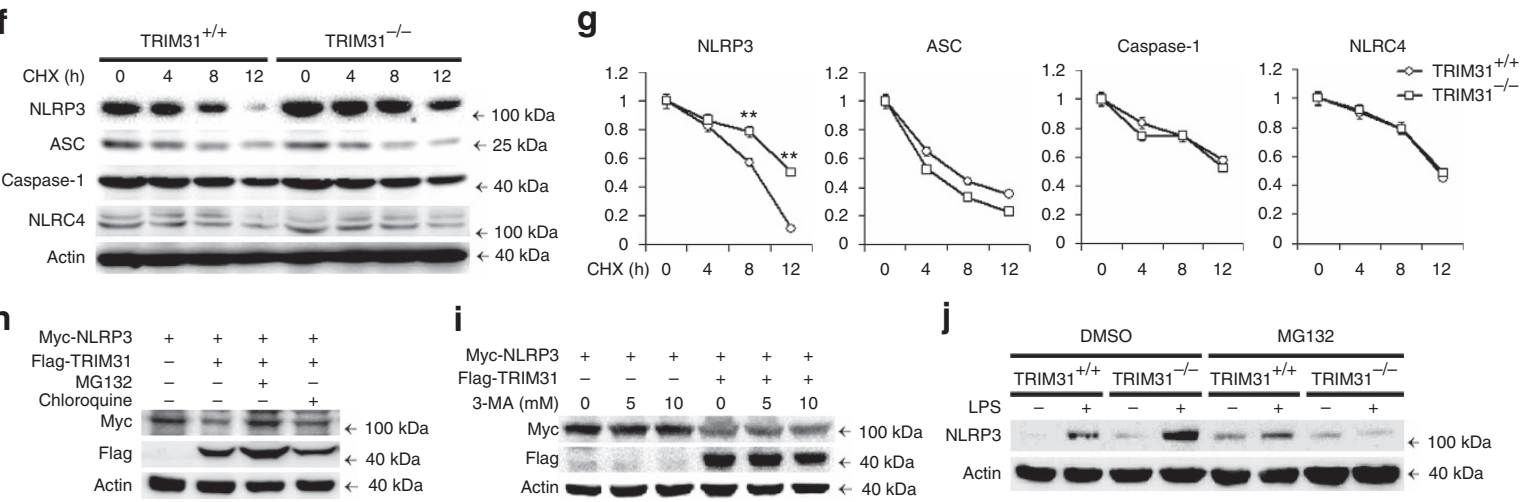

i
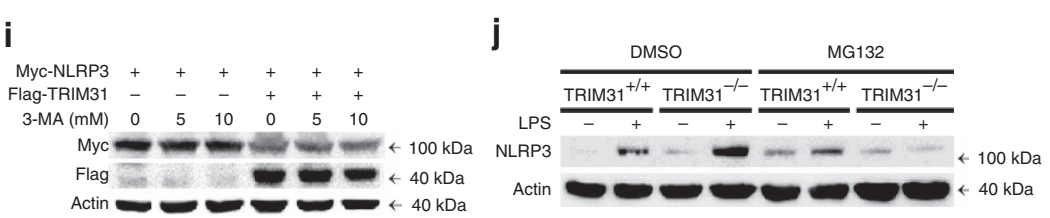

Figure 2 | TRIM31 promotes proteasomal degradation of NLRP3. (a) Immunoblot analysis of extracts (upper panel) or RT-PCR analysis (lower panel) of mouse peritoneal macrophages silenced of TRIM31. (b) Immunoblot analysis of extracts (upper panel) or RT-PCR analysis (lower panel) of mouse peritoneal macrophages silenced of TRIM31, then stimulated for various times with LPS. (c) Immunoblot analysis of extracts from HEK293T cells transfected with increasing amount of TRIM31 expression plasmid. (d) Immunoblot analysis of extracts from TRIM31 ${ }^{+/+}$or TRIM31-/ mouse peritoneal macrophages, then stimulated for various times with LPS. (e) Immunoblot analysis of NLRP3 expression from TRIM31+/+ or TRIM31- /mouse peritoneal macrophages, then stimulated with LPS, IL-1 $\beta$, poly(I:C), TNF- $\alpha$ or PGN for $8 \mathrm{~h}$. (f,g) Immunoblot analysis of extracts from TRIM31 $+/+$ or TRIM31-/ - mouse peritoneal macrophages stimulated with LPS for $4 \mathrm{~h}$, and then treated for various times with cycloheximide (CHX). NLRP3, ASC, Caspase- 1 and NLRC4 expression levels were quantitated by measuring band intensities using 'ImageJ' software. The values were normalized to actin (g). ${ }^{\star \star} P<0.01$. (mean and s.d. of three samples in $\mathbf{g}$, Student's $t$-test). (h) Immunoblot analysis of extracts from HEK293T cells transfected with Myc-NLRP3 and Flag-TRIM31 expression plasmid then treated with MG132 $(10 \mu \mathrm{M})$ or chloroquine $(10 \mu \mathrm{M})$ for $4 \mathrm{~h}$. (i) Immunoblot analysis of extracts from HEK293T cells transfected with Myc-NLRP3 and Flag-TRIM31 expression plasmid then treated with 3-MA as indicated for $4 \mathrm{~h}$. (j) Immunoblot analysis of NLRP3 expression from TRIM $31^{+/+}$or TRIM31-/- mouse peritoneal macrophages stimulated with LPS for $4 \mathrm{~h}$, together with DMSO or MG132 (10 $\mu$ M) treatment for $4 \mathrm{~h}$. Similar results were obtained in three independent experiments. 
plasmid (Fig. 4b), indicating TRIM31 mediated K48-linked polyubiquitination of NLRP3.

Under physiological conditions, the endogenous NLRP3 was observed to be robustly ubiquitinated with both K48 and K63 linkage upon LPS stimulation (Fig. 4c). However, only K48-linked polyubiquitination of NLRP3 was almost completely abolished in TRIM31 deficient macrophages, and K63-linked ubiquitination of NLRP3 was not affected (Fig. 4c). To determine whether TRIM31 directly modified NLRP3, we reconstituted the NLRP3ubiquitination reaction in vitro. We prepared recombinant NLRP3, recombinant TRIM31 (wt and $\triangle$ RING), purified E2 enzyme UbcH5A, a mixture of E1 plus ubiquitin (wt, K48 only and K63 only). We observed ubiquitination of NLRP3 only when TRIM31 wt was present (Fig. 4d). TRIM31 $\Delta$ RING lost the ability of promoting NLRP3 ubiquitination. Furthermore, we found ubiquitination of NLRP3 was mediated by K48 linkage but not by K63 linkage (Fig. 4d). Taken together, these data indicated that TRIM31 directly induced the K48-linked ubiquitination of NLRP3 through its E3 ligase activity.

K48-linked polyubiquitination leads to the degradation of target proteins through $26 \mathrm{~S}$ proteasome ${ }^{28}$. TRIM31 promoted proteasomal degradation of NLRP3 (Fig. 2g). However, TRIM31 mutants $\triangle$ RING and C16AC36A with the deprivation of the E3 ubiquitin ligase activity lost the ability to promote degradation of NLRP3 (Fig. 4e-g). All together, these data demonstrated that TRIM31 mediated K48-linked ubiqutination and proteasomal degradation through its E3 ubiquitin ligase activity.

LPS and IL-1 $\beta$ induce TRIM31 expression. Having established the inhibitory role of TRIM31 in NLRP3 inflammasome activation, we next examined the expression status of TRIM31 during the process of NLRP3 inflammasome activation. LPS or
IL-1 $\beta$ stimulation greatly induced TRIM31 expression at both mRNA and protein level (Fig. 5a,b).

TRIM31 deficiency enhances IL-1 $\beta$ secretion in vivo. We next investigated the biological effects of TRIM31 on NLRP3 inflammasome activation in vivo. The induction of IL- $1 \beta$ by intraperitoneal (i.p.) injection of LPS is NLRP3 dependent ${ }^{29,30}$. We then examined whether TRIM31 deficiency could regulate this induction of IL-1 $\beta$. TRIM31 deficiency enhanced serum concentrations of IL-1 $\beta$ but did not considerably increase the amount of TNF- $\alpha$ and IL-6 (Fig. 6a), indicating that TRIM31 could specifically attenuate NLRP3 inflammasome activation in vivo.

TRIM31 deficiency aggravates alum-induced peritonitis. We further investigated the effects of TRIM31 in an IL-1-dependent mouse peritonitis model ${ }^{31}$. Mouse peritonitis was induced by i.p. injection of alum. Peritoneal exudate cells (PECs) were collected and alum-induced recruitment of inflammatory cells was analysed by flow cytometry. Alum challenge greatly upregulated caspase-1 cleavage in the lavage fluid and that this was significantly enhanced by TRIM31 deficiency (Fig. 6b), which was consistent with the observation in vitro. In addition, more NLRP3 expression was also observed in the PECs lysates from TRIM31 deficient mice (Fig. 6b). We consequently analysed alum-induced recruitment of inflammatory cells in the lavage fluid. Alum-induced recruitment of neutrophils and $\mathrm{Ly}^{+} \mathrm{C}^{+}$ monocytes was strongly enhanced in the TRIM31 deficient mice (Fig. 6c). Collectively, these findings further demonstrated that TRIM31 could inhibit NLRP3 inflammasome activity and subsequent immune cell accumulation in mouse peritonitis in vivo.

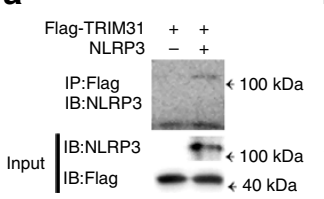

d TRIM31-GFP

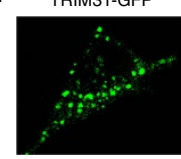

g
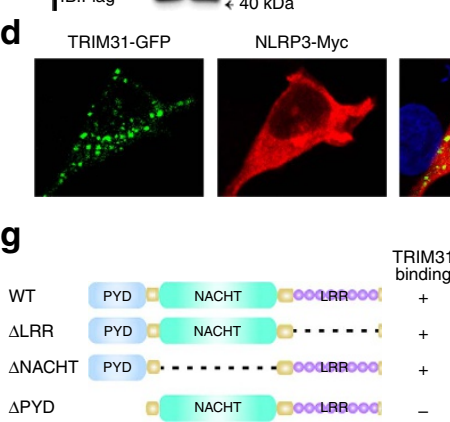

b

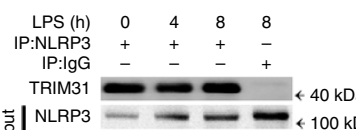

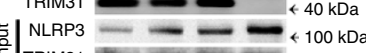

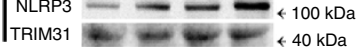
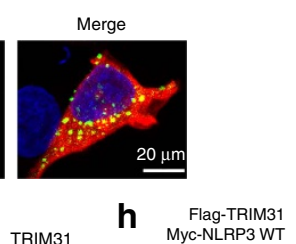
Myc-NLRP3 WT
Myc-NLRP3 $\triangle$ LRR
Myc-NLRP3 $\triangle$ NACHT
Myc-NLRP3 $\triangle$ PYD

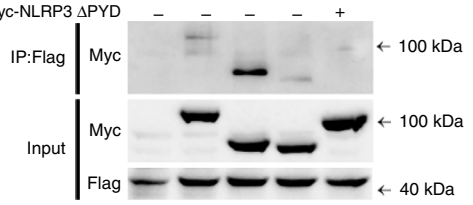

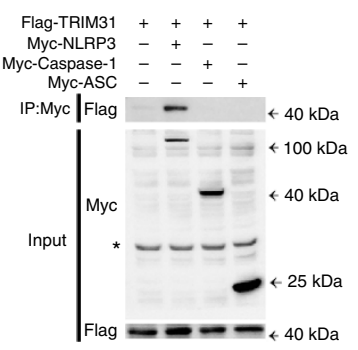

e

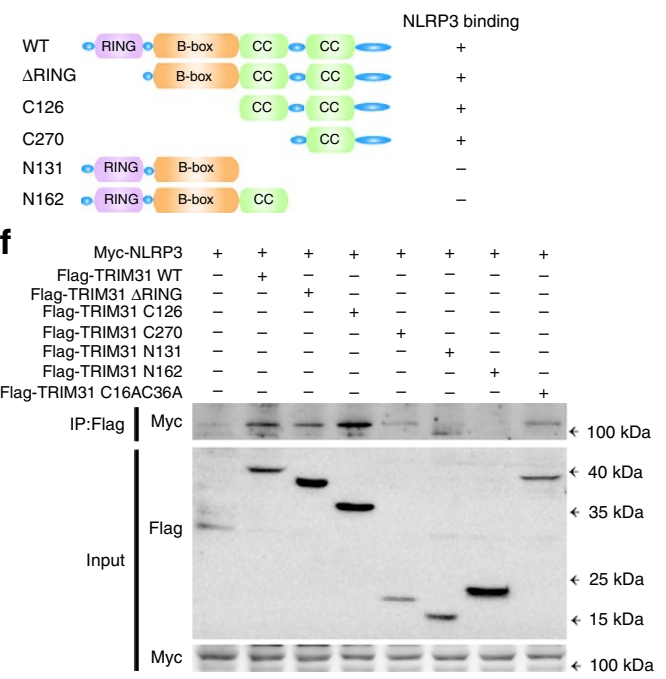

Figure 3 | TRIM31 interacts with NLRP3. (a) NLRP3 and Flag-tagged TRIM31 were obtained by in vitro transcription and translation. Interaction between TRIM31 and NLRP3 was assayed by mixing TRIM31 and NLRP3 together followed by IP with Flag antibody and immunoblot analysis with NLRP3 antibody. (b) Co-immunoprecipitation of endogenous TRIM31 with endogenous NLRP3 from mouse peritoneal macrophages stimulated with LPS for indicated time periods. (c) HEK293T cells expressing Flag-TRIM31 and Myc-NLRP3, Myc-Caspase-1 or Myc-ASC were lysed. Co-immunoprecipitation of Flag-TRIM31 with Myc-NLRP3 from HEK293T cells. *Non-specific band. (d) HEK293T cells transfected with GFP-TRIM31 and Myc-NLRP3 were fixed and incubated with a secondary antibody conjugated to Alexa Fluor 568. Colocalization between TRIM31 and NLRP3 was examined by Confocal microscopy. (e) Schematic diagram of TRIM31 and its truncation mutants. (f) Flag-tagged TRIM31 or its mutants and Myc-NLRP3 were individually transfected into HEK293T cells. The cell lysates were immunoprecipitated with an anti-Flag antibody and then immunoblotted with the indicated antibodies. (g) Schematic diagram of TRIM31 and its truncation mutants. (h) Myc-tagged NLRP3 or its mutants and Flag-TRIM31 were individually transfected into HEK293T cells. The cell lysates were immunoprecipitated with an anti-Flag antibody and then immunoblotted with the indicated antibodies. Similar results were obtained in three independent experiments. 
a

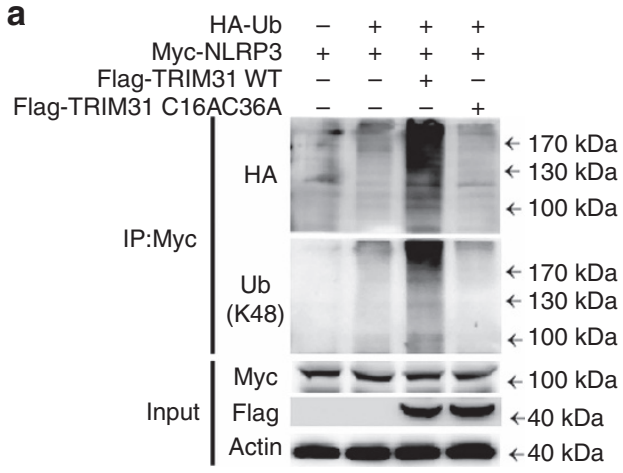

d

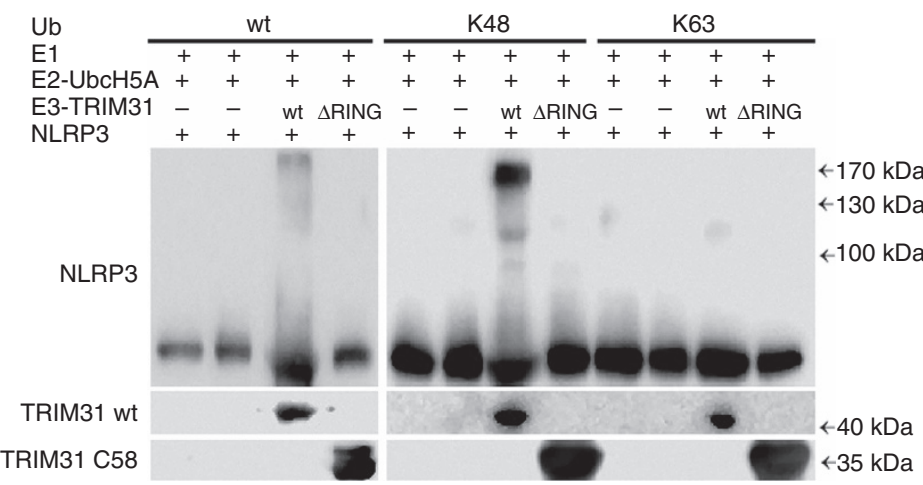

b

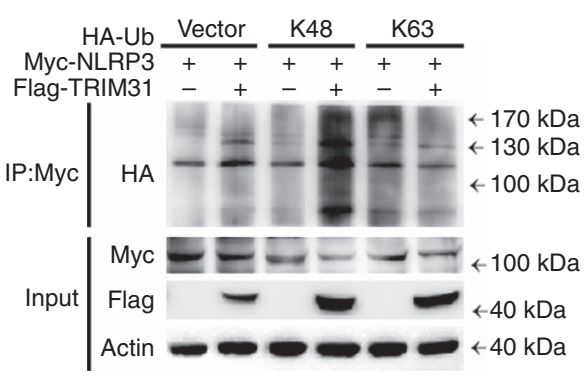

C

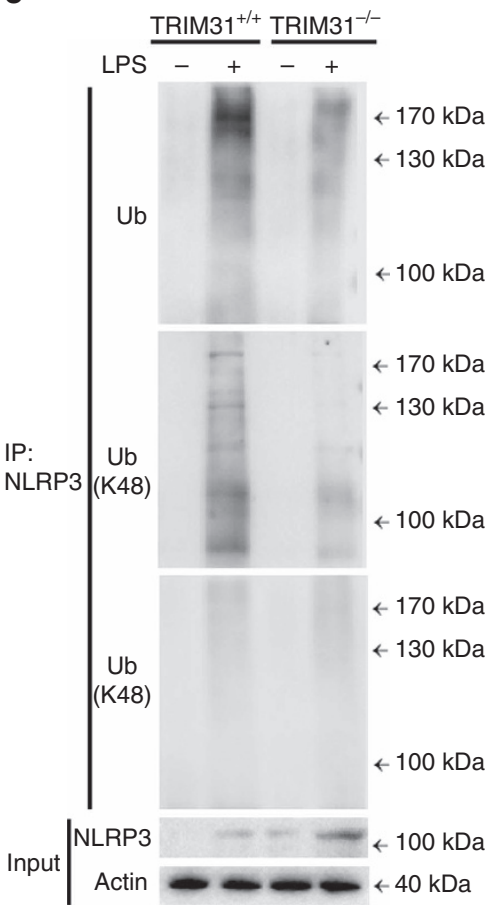

e

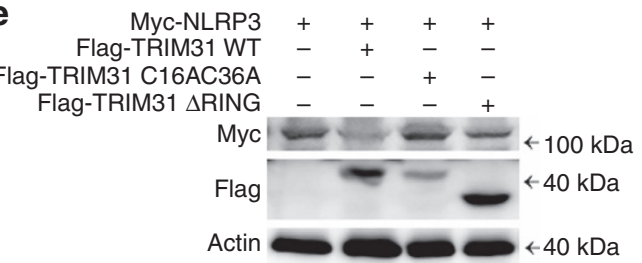

f

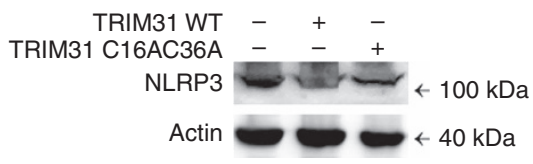

g

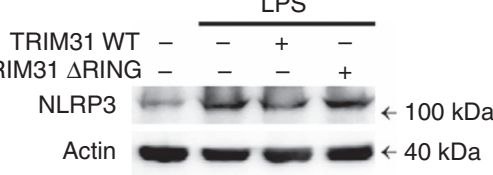

Figure 4 | TRIM31 promotes K48-linked polyubiquitination of NLRP3. (a) Immunoblot analysis of lysates from HEK293T cells transfected with HA-tagged ubiquitin (HA-Ub), Myc-NLRP3 and TRIM31 WT or C16AC36A, followed by IP with anti-Myc, probed with anti-HA or K48-Ub. (b) Immunoblot analysis of lysates from HEK293T cells transfected with HA-tagged K48-linked ubiquitin (K48-Ub) or HA-tagged K63-linked ubiquitin (K63-Ub), Myc-NLRP3 and TRIM31, followed by IP with anti-Myc, probed with anti-HA. (c) Immunoblot analysis of lysates from TRIM31+/+ or TRIM31-/ - mouse peritoneal macrophages, followed by IP with anti-NLRP3, probed with anti-Ub, K48-Ub or K63-Ub. (d) In vitro ubiquitination assay was performed in the presence of Ub (wt, K48 or K63), E1, E2-UbcH5A, NLRP3 and TRIM31 (wt or $\triangle R$ ING mutant). The ubiquitination of NLRP3 was examined with NLRP3 antibody. (e) Immunoblot analysis of extracts from HEK293T cells transfected with Myc-NLRP3 and Flag-tagged TRIM31 or its mutants. (f) Immunoblot analysis of extracts from THP-1 cells transfected with TRIM31 wild type (WT) or TRIM31 C16AC36A. (g) Immunoblot analysis of extracts from THP-1 cells transfected with TRIM31 WT or TRIM31 $\triangle$ RING then stimulated with LPS for $4 \mathrm{~h}$. Similar results were obtained in three independent experiments.
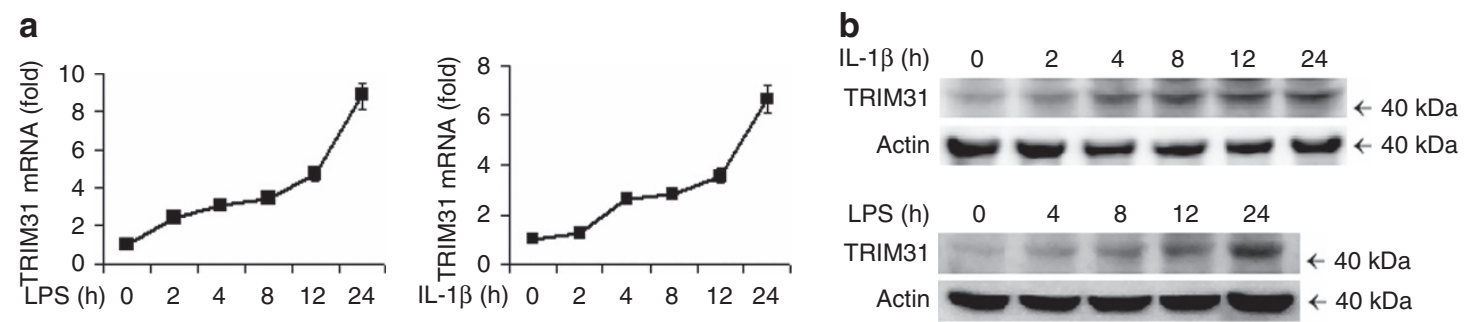

Figure 5 | IL-1; and LPS induce TRIM31 expression. (a,b) RT-PCR analysis (a) or immunoblot analysis (b) of TRIM31 expression from mouse peritoneal macrophages stimulated with LPS or IL-1 $\beta$ for various times. Similar results were obtained in three independent experiments. Data are representative of three experiments (mean and s.d. of six samples in a).

TRIM31 deficiency ameliorates DSS-induced colitis. DSS model is one of the most extensively used to investigate innate immune mechanisms of colitis ${ }^{32}$. NLRP3 inflammasome is critically involved in the maintenance of intestinal homeostasis and protection against colitis ${ }^{33}$. Besides spleen, TRIM31 expressed extremely high in gut, including small intestine and colon (Supplementary Fig. 5), indicating the functions of TRIM31 in maintaining intestinal homeostasis. We thus examined the potential roles of TRIM31 in the DDS-induced colitis model. To study the contribution of TRIM31 to the development of 
colitis, we first assessed the clinical features of age- and sex-matched WT and TRIM31 deficient mice after oral administration of $3 \%$ DSS in drinking water. TRIM31-/mice suffered from less body weight loss from day 4 on (Fig. 7a). Simultaneously, rectal bleeding scores and stool consistency scores of TRIM $31^{-/}$mice were significantly attenuated compared with those of DSS-fed WT mice
(Fig. 7b,c). To further assess the severity of colitis, colon length was measured in DSS-fed WT and TRIM $31^{-l-}$ mice. Colons of TRIM $31^{-1-}$ mice were longer than those of WT mice administered with DSS (Fig. $7 \mathrm{~d}, \mathrm{e}$ ). The severity of colonic inflammation and ulceration was further evaluated by histopathological analysis using haematoxylin \& eosin (H\&E) staining. DSS-induced mucosal damage was characterized by
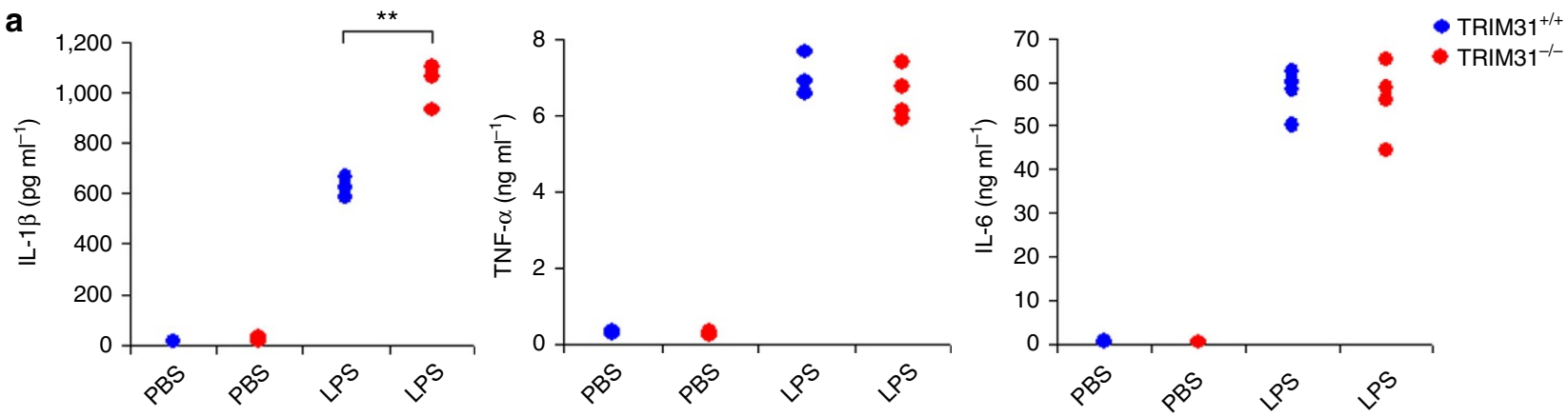

b

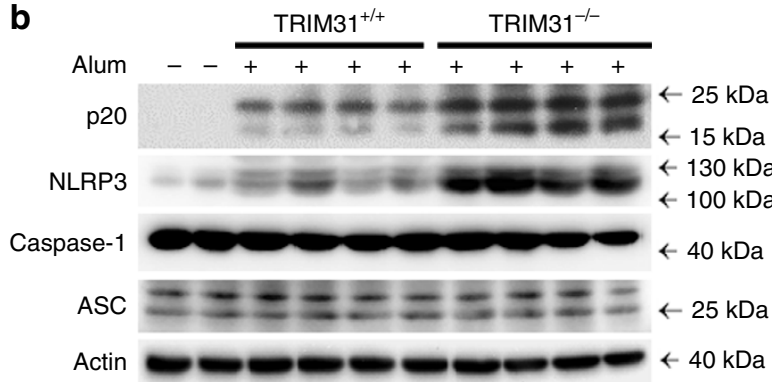

C

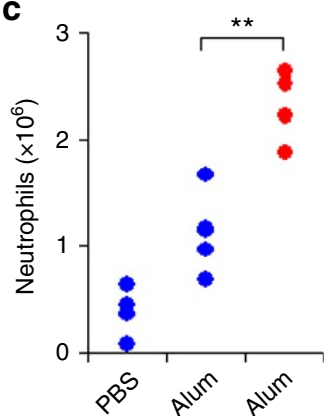

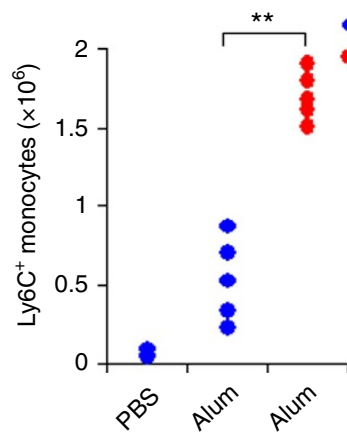

Figure 6 | TRIM31 deficiency enhances IL-1 $\beta$ secretion and aggravates Alum-induced peritonitis in vivo. (a) ELISA analysis of serum levels of IL-1 $\beta$,

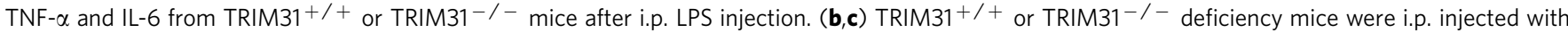
alum for $12 \mathrm{~h}$. PECs were lysed and analysed for the expression of Caspase-1, NLRP3 and ASC by immunoblot (b). Absolute numbers of neutrophils or Ly6C ${ }^{+}$monocytes recruited to the peritoneum were analysed by fluorescence-activated cell sorting (five mice per group) (c). ${ }^{\star \star} P<0.01$. (Student's $t$-test). Data are representative of three experiments (mean and s.d. of four to five samples in $\mathbf{a}$ and $\mathbf{c}$ ).
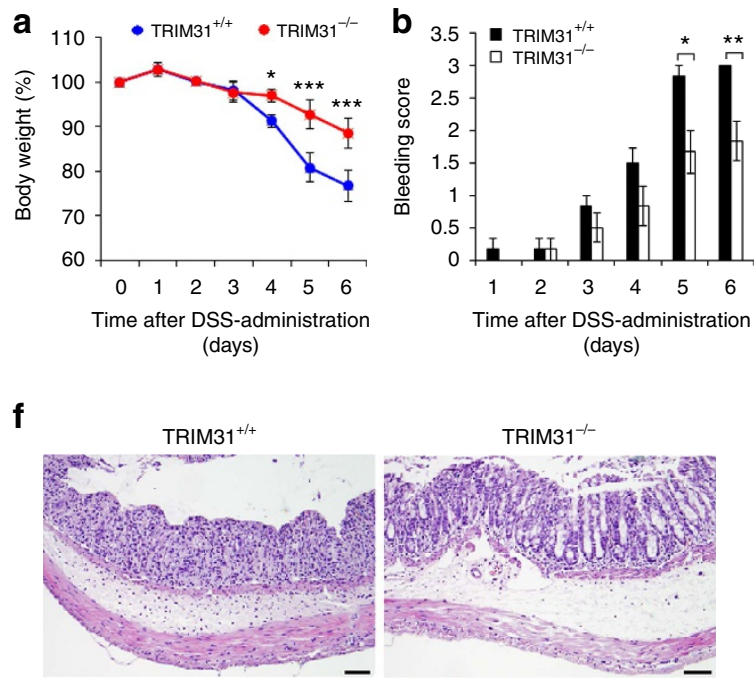
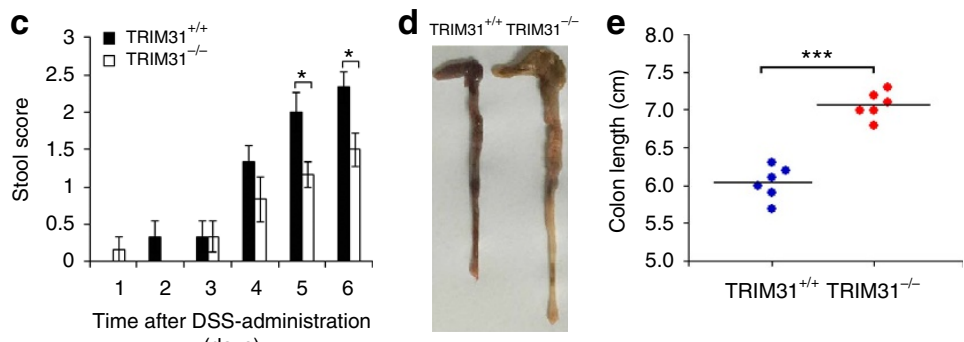

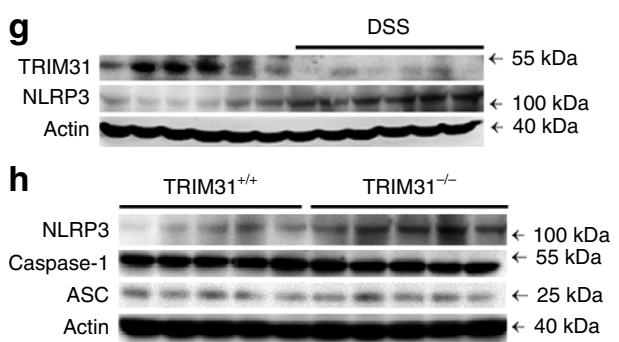

Figure 7 | TRIM31 deficiency ameliorates DSS-induced colitis. Mice were given 3\% DSS in their drinking water for 5 days, followed by regular drinking water. (a) Body weight, (b) stool consistency and (c) rectal bleeding score were scored daily. (d-h) Mice were killed on day 6. Macroscopic appearances (d) and colon lengths (e) of the mice were measured. Histopathological changes in colon tissue were examined by H\&E staining (f) Scale bars, $50 \mu \mathrm{m}$. Immunoblot analysis of lysates from colon tissue $(\mathbf{g}, \mathbf{h}) .{ }^{\star} P<0.05,{ }^{\star \star} P<0.01,{ }^{\star \star \star} P<0.001$ (one-way analysis of variance, ANOVA). 

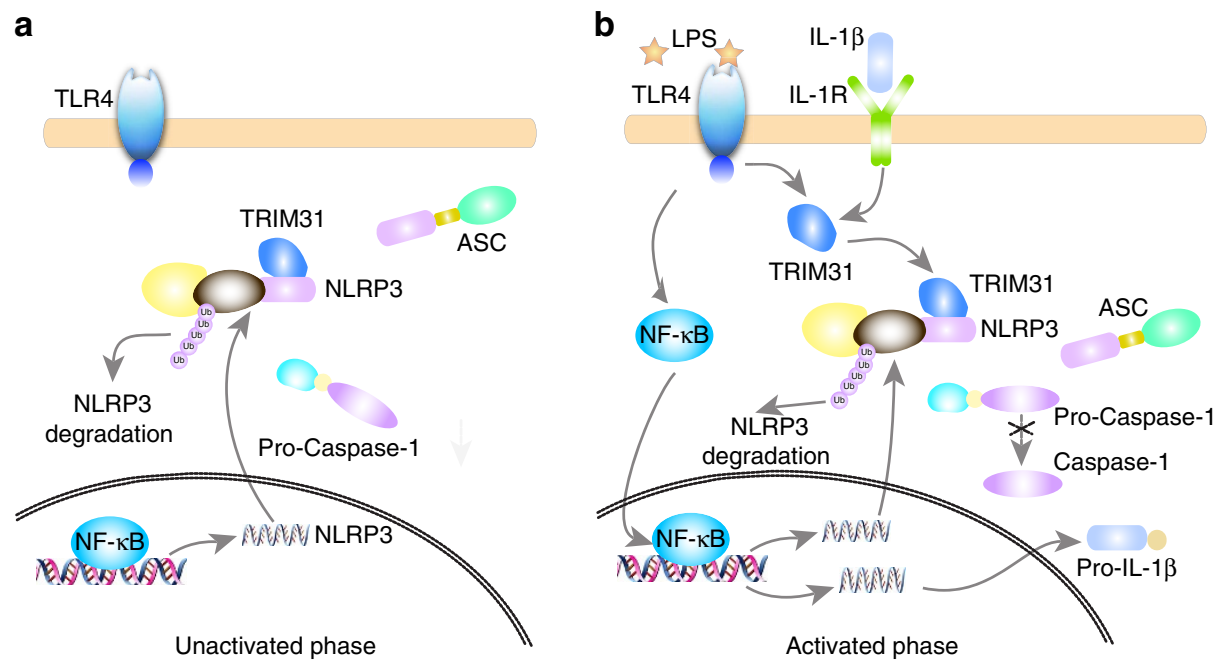

Figure 8 | Working model for TRIM31 inhibiting NLRP3 inflammasome activation. (a) In resting macrophages, the constitutively expressed TRIM31 binds to NLRP3, promotes K48-linked ubiquitination and proteasomal degradation of NLRP3, and maintains its low expression. (b) Following NLRP3 inflammasome activation, TRIM31 expression is markedly induced by LPS and IL-1 $\beta$, resulting in the inhibition of NLRP3 expression and subsequent inflammasome activation.

crypt loss and infiltrating leucocytes. Colonic sections of DSS-fed WT mice displayed severe transmural inflammation with focal areas of extensive ulceration and necrotic lesions (Fig. 7f). Inflammatory infiltrates filled the lamina propria and submucosa in areas where the mucosa was intact and often effaced the normal architecture of the tissue (Fig. 7f). Strikingly, colonic sections of DSS-fed TRIM31- ${ }^{-}$mice exhibited less inflammatory cell infiltration and intact colonic architecture with no apparent ulceration (Fig. 7f).

To confirm the roles of TRIM31 in the model, we examined TRIM31 and NLRP3 expressions in colon tissues. In WT mice, TRIM31 constitutely expressed in colon tissues (Fig. 7g, Supplementary Fig. 5), and that maintains NLRP3 protein expression in a relatively low level (Fig. $7 \mathrm{~g}$ ). Following DSS treatment, TRIM31 was downregulated and NLRP3 protein expression was elevated (Fig. 7g). In DSS-administered TRIM31 $1^{-1-}$ mice, NLRP3 protein expression in colon tissues was further enhanced (Fig. 7h). Collectively, these data indicated that TRIM31 played crucial roles in limiting NLRP3 expression and thus exacerbated DSS-induced colitis.

\section{Discussion}

Ubiqutination is a crucial post-transcriptional modification to modulate the intensity of innate immune responses, including regulation of NLRP3 inflammasome activity. For example, K63-linked ubiquitination of ASC targets the AIM2-ASC inflammasome complex for destruction in autophagosomes ${ }^{34,35}$. The linear ubiquitin assembly complex-mediated ASC linear ubiquitination is required for NLRP3 inflammasome activation ${ }^{36}$. A20 restricts ubiquitination of pro-interleukin- $1 \beta$ protein complexes to suppress NLRP3 inflammasome activity ${ }^{37}$. TRIM33 ubiquitinates DHX33 and is essential for the cytosolic RNA-induced NLRP3 inflammasome activation ${ }^{38}$. The deubiquitination of NLRP3 by the BRCC3 complex is required for NLRP3 inflammasome activation ${ }^{13,39,40}$. E3 ubiquitin ligase MARCH7 promoted NLRP3 K48-linked ubiquitination and subsequent degradation in an autophagy-dependent pathway ${ }^{18}$. However, whether the ubiquitinated NLRP3 could be degraded via a ubiquitin-proteasome pathway is still unknown. In the present study, we found that NLRP3 could be ubiquitinated with K48 linkage in both resting and activated macrophages, and E3 ubiquitin ligase TRIM31 mediated this modification. Thus, TRIM31 promoted proteasomal degradation of NLRP3 and then inhibited the NLRP3 inflammasome activation. Collectively, several E3 ubiquitin ligases, such as TRIM31, MARCH7, DHX33 and so on, could negatively or positively regulate NLRP3 inflammasome activation at different levels and via different mechanisms. As for the regulation of NLRP3 expression, E3 ubiquitin ligase TRIM31 and MARCH7 could promote the degradation of NLRP3 via a ubiquitin-proteasome pathway and autophagy-dependent pathway, respectively. Thus, the E3 ubiquitin ligases could work together to fine tune the activation of NLRP3 inflammasome to maintain immune homeostasis. Our research identify TRIM31 as a physiological regulator of NLRP3 expression, and that will further explain the complexities of homeostatic control of innate immune signalling.

Based on the experimental data, we propose a model to illustrate how TRIM31 feedback negatively regulates NLRP3 inflammasome activation (Fig. 8). In resting macrophages, the constitutively expressed TRIM31-mediated proteasomal degradation of NLRP3 protein to maintain its low expression and limit the NLRP3 inflammasome assembly (Fig. 8a). Following LPS stimulation, NLRP3 expression is induced rapidly through NF- $\kappa B$ activation, which could facilitate NLRP3 inflammasome assembly and activation. Simultaneously, TRIM31 expression is induced by LPS stimulation and IL- $1 \beta$ secretion. TRIM31 then directly bound to NLRP3 and promoted its K48-linked ubiquitination, leading to its proteasomal degradation (Fig. 8b). Thus, the activation of NLRP3 inflammasome was limited in a proper intensity and time course to avoid detrimental effects.

Improper NLRP3 inflammasome activation has been linked to a variety of diseases ${ }^{5}$, such as infectious diseases, autoinflammatory and autoimmune diseases. NLRP3 inflammasome activity is critical for host response to microbial pathogens. Excessive NLRP3 inflammasome activation plays crucial causative or contributing roles in the initial of autoinflammatory and autoimmune diseases ${ }^{5}$. However, optimal NLRP3 inflammasome activation is beneficial for some inflammatory disorders, such as inflammatory bowel diseases ${ }^{33}$. Thus, a better understanding of the balance between beneficial and detrimental NLRP3 inflammasome activation is needed.

In the present study, we found that TRIM31 constitutely expressed in macrophages and negatively regulated NLRP3 
inflammasome activation to prevent unwanted activation in both resting and activation state. In vivo experiments also confirmed the beneficial roles of TRIM31 in the alum-induced peritonitis, an NLRP3 dependent acute inflammatory model. Furthermore, TRIM31 also constitutively expressed in gut, limited NLRP3 expression in a relatively low level and thus possessed potential functions in maintaining intestinal homeostasis. However, TRIM $31^{+1+}$ mice were more susceptible to acute DSS-induced colitis, compared with TRIM $31^{-/-}$mice. Recent evidence has indicated that NLRP3 confers protection against acute colitis $^{33,41,42}$. TRIM31 expression was downregulated in the DSS-induced colitis model, and NLRP3 expression was upregulated accordingly. Thus, NLRP3 could exert its protection roles when colitis was initiated. In TRIM31-/mice, NLRP3 expression was further increased in the colon of DSS-induced colitis model. So, TRIM31 deficiency ameliorates DSS-induced colitis.

In summary, we identified TRIM31 as a feedback suppressor of NLRP3 inflammasome activity. Because of the conserved biological function of NLRP3 inflammasome in multiple physiological processes, fine-tuning of its activity is critical for the disease resistance and maintenance of immune homeostasis. Our research provides an explanation on the limitation of NLRP3 inflammasome activation under physiological conditions. Furthermore, TRIM31 may provide a useful therapeutic target for the intervention of diseases with improper inflammasome activation, such as autoinflammatory diseases, colitis, obesity and diabetes.

\begin{abstract}
Methods
Mice. TRIM $31^{-1-}$ mice on a C57BL/6J background were generated by Cyagen Biosciences Inc. (Guangzhou, China) using TALEN technology. A pair of TALEN constructs for TRIM31 knockout (KO) were cloned into a mammalian expression vector pCMV-TALEN and capped, polyA-tailed mRNA for injection were produced using the Ambion mMessage mMachine kit. The KO mice were produced by microinjecting TALEN mRNAs into fertilized eggs from C57BL/6 strain. The $\mathrm{KO}$ alleles have been sequence validated. Three kinds of $\mathrm{KO}$ mice were generated and named as $\mathrm{KO} 1, \mathrm{KO} 2$ and $\mathrm{KO} 3$. $\mathrm{KO} 1$ has one missing base pairs (C, 97 of the ORF), KO2 has one missing base pairs (G, 94 of the ORF) and $\mathrm{KO} 3$ has five missing base pairs (GGGCA, 94-98 of the ORF). All the three KOs caused a frame shift. The mRNA transcribed from targeted allele with frame shift undergoes nonsense-mediated decay. $\mathrm{KO} 1$ mice were used in most experiments if no special noted. The mice were backcrossed with C57BL/6 mice for more than six generations. C57BL/6 mice were obtained from Vital River Laboratory Animal Technology Co. (Beijing, China). All animal experiments were undertaken in accordance with the National Institute of Health Guide for the Care and Use of Laboratory Animals, with the approval of the Scientific Investigation Board of Medical School of Shandong University (Jinan, Shandong Province, China).
\end{abstract}

Reagents. ATP, MG132, chloroquine, cycloheximide, phorbol myristate acetate, LPS (Escherichia coli, 055:B5), peptidoglycan (PGN), poly(I:C), 3-MA, anti-TRIM31, anti-Myc and anti-Flag were from Sigma-Aldrich (St Louis, MO); Dextran sulfate sodium (DSS, 36-50 kDa) was bought from MP Biomeicals (Aurora, OH). Imject Alum was from Thermo Scientific; IL-1 $\beta$ and TNF- $\alpha$ were from PeproTech (Rocky Hill, NJ); Flagellin and poly(dA:dT) were from Invivogen (San Diego, CA). Nigericin, anti-caspase-1 p45\&p20, anti-NLRP3 and anti-ASC were from AdipoGen; anti-hemagglutinin (HA), anti-Ub, anti- $\beta$-actin, protein $G$ agarose used for IP and horseradish peroxidase-conjugated secondary antibodies were from Santa Cruz Biotechnology (Santa Cruz, CA); anti-IL-1 $\beta$ p31\&p17, anti-p-JNK, anti-p-p38, anti-p-ERK, anti-JNK, anti-p38, anti-ERK1/2, anti-p-IкB $\alpha$, anti-K48-ub and anti-K63-ub were from Cell Signaling Technology (Beverly, MA); anti-AIM2 and anti-NLRC4 were from Abcam (Cambridge, MA).

Cell culture. To obtain mouse primary peritoneal macrophages, C57BL/6J mice (female, $4-6$ weeks old) were injected intraperitoneally with $3 \%$ Brewer's thioglycollate broth. Three days later, PEC were harvested and incubated. Two hours later, nonadherent cells were removed and the adherent monolayer cells were used as peritoneal macrophages ${ }^{14,40,43}$. Human THP-1 and human embryonic kidney (HEK293T) cells were obtained from American Type Culture Collection (Manassas, VA). Phorbol myristate acetate-activated THP-1 cells were used as human macrophages. The cells were cultured at $37^{\circ} \mathrm{C}$ under $5 \% \mathrm{CO}_{2}$ in DMEM supplemented with 10\% FCS (Invitrogen-Gibco), $100 \mathrm{U} \mathrm{ml}^{-1}$ penicillin, and $100 \mu \mathrm{g} \mathrm{ml}^{-1}$ streptomycin. The concentration of agonists or stimuli were used as below: LPS $100 \mathrm{ng} \mathrm{ml}^{-1}$ for mouse primary peritoneal macrophages, LPS $1 \mu \mathrm{g} \mathrm{ml}^{-1}$ for THP-1 cells, ATP $5 \mathrm{mM}$, nigericin $50 \mu \mathrm{M}$ and alum $200 \mu \mathrm{g} \mathrm{ml}^{-1}$, IL-1 $\beta 10 \mathrm{ng} \mathrm{ml}^{-1}$, poly(I:C) $10 \mu \mathrm{g} \mathrm{ml}^{-1}$, TNF- $\alpha 200 \mathrm{ng} \mathrm{ml}^{-1}$ and PGN $5 \mu \mathrm{g} \mathrm{ml}^{-1}$. Poly (dA:dT) and flagellin were transfected into macrophages with the final concentration as below: poly(dA:dT) $200 \mathrm{ng} \mathrm{ml}^{-1}$ and flagellin $200 \mathrm{ng} \mathrm{ml}^{-1}$.

Plasmids construction and transfection. TRIM31 expression plasmid was constructed by PCR-based amplification of cDNA from THP-1 cells, and then cloned into the pFLAG-CMV-2 eukaryotic expression vector (Sigma-Aldrich). Caspase-1 expression plasmid was constructed by PCR-based amplification of cDNA from THP-1 cells, and then cloned into the pCMV-Myc eukaryotic expression vector (Beyotime, China). Myc-NLRP3 and Myc-ASC plasmids were provided by Dr John C. Reed (Sanford-Burnham Medical Research Institute, La Jolla, CA). Expression vectors for HA-Ub WT, mutant K48 and mutant K63 were from Dr Hui Xiao (Institut Pasteur of Shanghai, CAS, Shanghai, China). MyD88 plasmid was gifts from Dr Xuetao Cao (Second Military Medical University, Shanghai, China). NF- $\mathrm{KB}$ reporter plasmid was purchased from Stratagene. Truncated mutants of TRIM31 or NLRP3 were generated using the KOD-Plus-Mutagenesis kit (Toyobo, Osaka, Japan). All constructs were confirmed by DNA sequencing. Plasmids were transiently transfected into HEK293T cells or THP-1 cells with jetPRIME reagents (Polyplus) according to the manufacturer's instructions.

RNA interference assay. siRNAs were synthesized as following: murine TRIM31: 5'-GCUCACUAAAUCCUUGAAA-3', human TRIM31: 5'-GGACCACAAAUCCCAUAAU- $3^{\prime}$ and negative control: $5^{\prime}$-UUCUCCGAACGUGUCACGU- $3^{\prime}$. These siRNA duplexes were transfected into mouse peritoneal macrophages or THP-1 cells using INTERFERin reagents (PolyPlus) according to the manufacturer's instructions.

ELISA. The concentrations of mouse IL-1 $\beta$, mouse TNF- $\alpha$ and mouse IL- 6 were measured using ELISA kits (Dakewe Biotech Company Ltd., Shenzhen, China) according to the manufacturer's instruction.

RNA quantitation. Total RNA was extracted with TRIzol reagent according to the manufacturer's instructions (Invitrogen). A LightCycler (ABI PRISM 7000) and a SYBR RT-PCR kit (Takara) were used for quantitative real-time RT-PCR analysis. Specific primers used for RT-PCR assays were the sequences of primers used for RT-PCR were $5^{\prime}$-TGGATGGGTTTGCTGGGAT- $3^{\prime}, 5^{\prime}$-CTGCGTGTAGCGACTG TTGAG- $3^{\prime}$ for NLRP3; $5^{\prime}$-ACCTTCCAGGATGAGGACATGA-3' ${ }^{\prime} 5^{\prime}$-AACGTCA CACACCAGCAGGTTA-3' for IL-1 $\beta$; $5^{\prime}$-CCAGAGTCAAACCGTGAGCG- $3^{\prime}$, $5^{\prime}$-GGCAACTTGGAGCCCGAA-3' for TRIM31; $5^{\prime}$-GCCACCACGCTCTTCTG TCT- $3^{\prime}, 5^{\prime}$-TGAGGGTCTGGGCCATAGAAC- $3^{\prime}$ for TNF- $\alpha$; and $5^{\prime}$-TGTTACC AACTGGGACGACA' $3,5^{\prime}$-CTGGGTCATCTTTTCACGGT- $3^{\prime}$ for $\beta$-actin. Data are normalized to $\beta$-actin expression in each sample.

Immunoprecipitation and immunoblot analysis. For IP, whole-cell extracts were lysed in IP buffer containing $1.0 \%$ (vol/vol) Nonidet P 40,50 mM Tris- $\mathrm{HCl}$, pH 7.4, $50 \mathrm{mM}$ EDTA, $150 \mathrm{mM} \mathrm{NaCl}$, and a protease inhibitor 'cocktail' (Merck). After centrifugation for $10 \mathrm{~min}$ at $14,000 \mathrm{~g}$, supernatants were collected and incubated with protein $\mathrm{G}$ Plus-Agarose Immunoprecipitation reagent together with specific antibody. After $6 \mathrm{~h}$ of incubation, beads were washed five times with IP buffer. Immunoprecipitates were eluted by boiling with $1 \%$ (wt/vol) SDS sample buffer. For immunoblot analysis, cells were lysed with M-PER Protein Extraction Reagent (Pierce, Rockford, IL) supplemented with a protease inhibitor 'cocktail', then protein concentrations in the extracts were measured with a bicinchoninic acid assay (Pierce, Rockford, IL). Equal amounts of extracts were separated by SDS-polyacrylamide gel electrophoresis (PAGE), and then were transferred onto nitrocellulose membranes for immunoblot analysis. Cell culture supernatants were harvested and concentrated for immunoblot with Amicon Ultra $10 \mathrm{~K}$ from Millipore. The concentration of primary antibodies used for immunoblot was $1 \mu \mathrm{g} \mathrm{ml}^{-1}$. The secondary antibodies were used 1 in 5,000. Uncropped scans of immunoblots are provided as Supplementary Figs 6-8.

In vitro binding and ubiquitination assay. NLRP3, TRIM31 WT and $\triangle$ RING mutant proteins were expressed with a TNT Quick Coupled Transcription/ Translation System (Promega) according to the instructions of the manufacturer ${ }^{22}$ Binding assays were performed by mixing Flag-tagged TRIM31 and NLRP3 together, followed by IP with Flag antibody and WB with NLRP3 antibody. Ubiquitination was analysed with an ubiquitination kit (Boston Biochem) following protocols recommended by the manufacturer.

Luciferase assay. Luciferase activities were measured with Dual-Luciferase Reporter Assay System (Promega) on a microplate luminometer (Centro LB 960; Berthold, Wildbad, Germany) according to the manufacturer's instructions. Data are normalized for transfection efficiency by subtracting Firefly luciferase activity with that of Renilla luciferase. 
Immunofluorescence staining and confocal analysis. HEK293T cells transiently transfected with plasmids encoding Myc-NLRP3 and GFP-TRIM31 were cultured for $24 \mathrm{~h}$. Myc-tagged NLRP3 was detected directly following fixation and washing. Anti-Myc Ab was used at 1:1,000 dilutions in the blocking solution. The cells were then incubated with Alexa Fluor 568-conjugated secondary Ab (Molecular Probes, Invitrogen) diluted 1:1,000 in blocking solution. Nuclei were stained with DAPI (4', 6'-diamidino-2-phenylindole hydrochloride; Molecular Probes, Invitrogen). Cells were examined with confocal laser microscopy (LSM780, Carl Zeiss, Oberkochen, Germany).

In vivo LPS challenge. WT or TRIM31 deficiency mice (females, 6 weeks old) were i.p. injected with $10 \mathrm{mg} \mathrm{kg}^{-1}$ LPS or PBS ${ }^{30}$. After $2 \mathrm{~h}$ mice were killed, and serum levels of IL-1 $\beta$, TNF- $\alpha$ and IL- 6 were measured by ELISA.

In vivo peritonitis. WT or TRIM31 deficiency mice (females, 6 weeks old) were i.p. injected with $700 \mu \mathrm{g}$ alum for $12 \mathrm{~h}$. Peritoneal cavities were washed with $6 \mathrm{ml}$ of PBS. The peritoneal fluids were harvested and concentrated for ELISA analysis with Amicon Ultra $10 \mathrm{~K}$ from Millipore. PECs were analysed by FACS ${ }^{14,31}$

DSS-induced colitis. Colitis was induced in WT or TRIM31 deficiency C57BL/6 mice (females, 6 weeks old) with 3\% (wt/vol) DSS dissolved in drinking water for 5 days, followed by regular drinking water. Mice were monitored for body weight, stool consistency and the presence of occult blood every day. Scoring for stool consistency and occult blood was done as described previously ${ }^{32,33}$. In brief, stool scores were determined as follows: 0 , well-formed pellets; 1 , semiformed stools that did not adhere to the anus; 2 , semiformed stools that adhered to the anus; 3 , liquid stools that adhered to the anus. Bleeding scores were determined as follows: 0 , no blood as tested with hemoccult (Beckman Coulter); 1, positive hemoccult; 2, blood traces in stool visible; 3 , gross rectal bleeding. After day 6 , the entire colon was excised to measure the length of the colon and the weight of caecum. Then $\sim 0.5 \mathrm{~cm}$ of mice colon tissues close to the rectum was collected for histological analysis and protein extraction. For histological analysis, formalin-fixed and paraffin-embedded segments of colon tissue were sectioned at $4 \mathrm{~mm}$ in thickness, and the sections were stained with hematoxylin and eosin (H\&E). TRIM31, NLRP3, Caspase-1 and ASC expression were examined with immunoblot analysis.

Statistical analysis. All experiments were independently performed three times. Data are presented as means \pm s.d. of three or four experiments. Analysis was performed using a Student's $t$-test or One-way analysis of variance (ANOVA) Values of $P<0.05$ were considered to be statistically significant.

Data availability. The data that support this study are available within the article and its Supplementary Information files or available from the authors upon request.

\section{References}

1. Schroder, K. \& Tschopp, J. The inflammasomes. Cell 140, 821-832 (2010).

2. Davis, B. K., Wen, H. \& Ting, J. P. The inflammasome NLRs in immunity, inflammation, and associated diseases. Annu. Rev. Immunol. 29, 707-735 (2011).

3. Rathinam, V. A., Vanaja, S. K. \& Fitzgerald, K. A. Regulation of inflammasome signaling. Nat. Immunol. 13, 333-342 (2012).

4. Latz, E., Xiao, T. S. \& Stutz, A. Activation and regulation of the inflammasomes. Nat. Rev. Immunol. 13, 397-411 (2013).

5. Guo, H., Callaway, J. B. \& Ting, J. P. Inflammasomes: mechanism of action, role in disease, and therapeutics. Nat. Med. 21, 677-687 (2015).

6. Strowig, T., Henao-Mejia, J., Elinav, E. \& Flavell, R. Inflammasomes in health and disease. Nature 481, 278-286 (2012).

7. Wen, H., Ting, J. P. \& O'Neill, L. A. A role for the NLRP3 inflammasome in metabolic diseases--did Warburg miss inflammation? Nat. Immunol. 13, 352-357 (2012)

8. Choi, A. J. \& Ryter, S. W. Inflammasomes: molecular regulation and implications for metabolic and cognitive diseases. Mol. Cells 37, 441-448 (2014).

9. Broderick, L. et al. The inflammasomes and autoinflammatory syndromes. Annu. Rev. Pathol. 10, 395-424 (2015).

10. Dowling, J. K. \& O'Neill, L. A. Biochemical regulation of the inflammasome. Crit. Rev. Biochem. Mol. Biol. 47, 424-443 (2012).

11. Haneklaus, M., O'Neill, L. A. \& Coll, R. C. Modulatory mechanisms controlling the NLRP3 inflammasome in inflammation: recent developments. Curr. Opin. Immunol. 25, 40-45 (2013).

12. Bauernfeind, F. G. et al. Cutting edge: NF-kappaB activating pattern recognition and cytokine receptors license NLRP3 inflammasome activation by regulating NLRP3 expression. J. Immunol. 183, 787-791 (2009).

13. Juliana, C. et al. Non-transcriptional priming and deubiquitination regulate NLRP3 inflammasome activation. J. Biol. Chem. 287, 36617-36622 (2012).
14. Huai, W. et al. Aryl hydrocarbon receptor negatively regulates NLRP3 inflammasome activity by inhibiting NLRP3 transcription. Nat. Commun. 5, 4738 (2014).

15. Bauernfeind, F. et al. NLRP3 inflammasome activity is negatively controlled by miR-223. J. Immunol. 189, 4175-4181 (2012).

16. Haneklaus, M. et al. Cutting edge: miR-223 and EBV miR-BART15 regulate the NLRP3 inflammasome and IL-1 $\beta$ production. J. Immunol. 189, 3795-3799 (2012).

17. Chuang, S. Y. et al. TLR-induced PAI-2 expression suppresses IL-1 $\beta$ processing via increasing autophagy and NLRP3 degradation. Proc. Natl Acad. Sci. USA 110, 16079-16084 (2013).

18. Yan, Y. et al. Dopamine controls systemic inflammation through inhibition of NLRP3 inflammasome. Cell 160, 62-73 (2015).

19. Versteeg, G. A., Benke, S., García-Sastre, A. \& Rajsbaum, R. InTRIMsic immunity: positive and negative regulation of immune signaling by tripartite motif proteins. Cytokine Growth Factor Rev. 25, 563-576 (2014).

20. Meyer, M., Gaudieri, S., Rhodes, D. A. \& Trowsdale, J. Cluster of TRIM genes in the human MHC class I region sharing the B30.2 domain. Tissue Antigens 61, 63-71 (2003).

21. Uchil, P. D. et al. TRIM protein-mediated regulation of inflammatory and innate immune signaling and its association with antiretroviral activity. J. Virol. 87, 257-272 (2013).

22. Wang, P., Zhao, W., Zhao, K., Zhang, L. \& Gao, C. TRIM26 negatively regulates interferon- $\beta$ production and antiviral response through polyubiquitination and degradation of nuclear IRF3. PLoS Pathog. 11, e1004726 (2015).

23. Suzuki, M. et al. TRIM39 negatively regulates the NFKB-mediated signaling pathway through stabilization of cactin. Cell Mol. Life Sci. 73, 1085-1101 (2016).

24. Zhao, W., Wang, L., Zhang, M., Yuan, C. \& Gao, C. E3 ubiquitin ligase tripartite motif 38 negatively regulates TLR-mediated immune responses by proteasomal degradation of TNF receptor-associated factor 6 in macrophages. J. Immunol. 188, 2567-2574 (2012).

25. Hu, M. M. et al. TRIM38 inhibits TNF $\alpha$ - and IL-1 $\beta$-triggered NF- $\mathrm{KB}$ activation by mediating lysosome-dependent degradation of TAB2/3. Proc. Natl Acad. Sci. USA 111, 1509-1514 (2014).

26. Cai, X. et al. Tripartite motif containing protein 27 negatively regulates CD4 T cells by ubiquitinating and inhibiting the class II PI3K-C2 $\beta$. Proc. Natl Acad. Sci. USA 108, 20072-20077 (2011).

27. Zheng, Q. et al. Siglecl suppresses antiviral innate immune response by inducing TBK1 degradation via the ubiquitin ligase TRIM27. Cell Res. 25, $1121-1136$ (2015).

28. Jiang, X. \& Chen, Z. J. The role of ubiquitylation in immune defence and pathogen evasion. Nat. Rev. Immunol. 12, 35-48 (2011).

29. He, Y., Franchi, L. \& Núñez, G. TLR agonists stimulate Nlrp3-dependent IL-1 $\beta$ production independently of the purinergic $\mathrm{P} 2 \mathrm{X} 7$ receptor in dendritic cells and in vivo. J. Immunol. 190, 334-339 (2013).

30. Coll, R. C. et al. A small-molecule inhibitor of the NLRP3 inflammasome for the treatment of inflammatory diseases. Nat. Med. 21, 248-255 (2015).

31. Guarda, G. et al. Type I interferon inhibits interleukin-1 production and inflammasome activation. Immunity 34, 213-223 (2011).

32. Wirtz, S., Neufert, C., Weigmann, B. \& Neurath, M. F. Chemically induced mouse models of intestinal inflammation. Nat. Protoc. 2, 541-546 (2007).

33. Zaki, M. H. et al. The NLRP3 inflammasome protects against loss of epithelial integrity and mortality during experimental colitis. Immunity 32, 379-391 (2010).

34. Shi, C. S. et al. Activation of autophagy by inflammatory signals limits IL-1 $\beta$ production by targeting ubiquitinated inflammasomes for destruction. Nat. Immunol. 13, 255-263 (2012).

35. Shimada, K. et al. Oxidized mitochondrial DNA activates the NLRP3 inflammasome during apoptosis. Immunity 36, 401-414 (2012).

36. Rodgers, M. A. et al. The linear ubiquitin assembly complex (LUBAC) is essential for NLRP3 inflammasome activation. J. Exp. Med. 211, 1333-1347 (2014).

37. Duong, B. H. et al. A20 restricts ubiquitination of pro-interleukin-1 $\beta$ protein complexes and suppresses NLRP3 inflammasome activity. Immunity 42, 55-67 (2015).

38. Weng, L. et al. The E3 ubiquitin ligase tripartite motif 33 is essential for cytosolic RNA-induced NLRP3 inflammasome activation. J. Immunol. 193, 3676-3682 (2014).

39. Lopez-Castejon, G. et al. Deubiquitinases regulate the activity of caspase-1 and interleukin-1 $\beta$ secretion via assembly of the inflammasome. J. Biol. Chem. 288, $2721-2733$ (2013).

40. Py, B. F., Kim, M. S., Vakifahmetoglu-Norberg, H. \& Yuan, J. Deubiquitination of NLRP3 by BRCC3 critically regulates inflammasome activity. Mol. Cell 49, 331-338 (2013).

41. Allen, I. C. et al. The NLRP3 inflammasome functions as a negative regulator of tumorigenesis during colitis-associated cancer. J. Exp. Med. 207, 1045-1056 (2010).

42. Hirota, S. A. et al. NLRP3 inflammasome plays a key role in the regulation of intestinal homeostasis. Inflamm. Bowel Dis. 17, 1359-1372 (2011). 
43. Zhang, M. et al. TRAF-interacting protein (TRIP) negatively regulates IFN- $\beta$ production and antiviral response by promoting proteasomal degradation of TANK-binding kinase 1. J. Exp. Med. 209, 1703-1711 (2012).

\section{Acknowledgements}

This work was supported by Grants from the National Natural Science Foundation of China (81622030, 31370017, 31570867, 81525012 and 81471538), Shandong Provincial Nature Science Foundation for Distinguished Young Scholars (JQ201420) and Key research and development program of Shandong Province (2015GSF118159).

\section{Author contributions}

W.Z. conceived the study, designed the experiments and provided overall direction; H.S., B.L., W.H., Z.Y and W.W. did the experiments; J.Z. did the artwork of the model figure; C.G. provided TRIM31 deficient mice; L.H., G.J. and L.Z. provided expertise and advice; H.S., B.L., W.H. and W.Z analysed the data and wrote the paper.

\section{Additional information}

Supplementary Information accompanies this paper at http://www.nature.com/ naturecommunications
Competing financial interests: The authors declare no competing financial interests.

Reprints and permission information is available online at http://npg.nature.com/ reprintsandpermissions/

How to cite this article: Song, H. et al. The E3 ubiquitin ligase TRIM31 attenuates NLRP3 inflammasome activation by promoting proteasomal degradation of NLRP3. Nat. Commun. 7, 13727 doi: 10.1038/ncomms13727 (2016).

Publisher's note: Springer Nature remains neutral with regard to jurisdictional claims in published maps and institutional affiliations.

\section{(c) (i)}

This work is licensed under a Creative Commons Attribution 4.0 International License. The images or other third party material in this article are included in the article's Creative Commons license, unless indicated otherwise in the credit line; if the material is not included under the Creative Commons license, users will need to obtain permission from the license holder to reproduce the material. To view a copy of this license, visit http://creativecommons.org/licenses/by/4.0/

(C) The Author(s) 2016 